\title{
Half-Duplex and Full-Duplex AF and DF Relaying with Energy-Harvesting in Log-Normal Fading
}

\author{
Khaled M. Rabie, Member, IEEE, Bamidele Adebisi, Senior Member, IEEE, Mohamed-Slim Alouini, Fellow, IEEE
}

\begin{abstract}
Energy-harvesting (EH) and wireless power transfer in cooperative relaying networks have recently attracted a considerable amount of research attention. Most of the existing work on this topic however focuses on Rayleigh fading channels, which represent outdoor environments. In contrast, this paper is dedicated to analyze the performance of dual-hop relaying systems with EH over indoor channels characterized by lognormal fading. Both half-duplex (HD) and full-duplex (FD) relaying mechanisms are studied in this work with decode-andforward (DF) and amplify-and-forward (AF) relaying protocols. In addition, three EH schemes are investigated, namely, time switching relaying, power splitting relaying and ideal relaying receiver which serves as a lower bound. The system performance is evaluated in terms of the ergodic outage probability for which we derive accurate analytical expressions. Monte Carlo simulations are provided throughout to validate the accuracy of our analysis. Results reveal that, in both HD and FD scenarios, AF relaying performs only slightly worse than DF relaying which can make the former a more efficient solution when the processing energy cost at the DF relay is taken into account. It is also shown that FD relaying systems can generally outperform HD relaying schemes as long as the loop-back interference in FD is relatively small. Furthermore, increasing the variance of the log-normal channel has shown to deteriorate the performance in all the relaying and $\mathrm{EH}$ protocols considered.
\end{abstract}

Index Terms-Amplify-and-forward relay, decode-and-forward relay, ergodic outage probability, full-duplex, half-duplex, energyharvesting protocols, log-normal fading, wireless power transfer.

\section{INTRODUCTION}

In conventional energy-constrained wireless networks, the network connectivity and operability is traditionally maintained from manually recharging or replacement of batteries which can be in many scenarios inconvenient or even impossible in others. Scavenging energy from the surrounding environment, also commonly known as energy harvesting (EH), using for instance solar power, thermal energy or wind energy seems to offer a promising and cost-effective solution that can prolong the life-time of energy-constrained wireless devices. However, such uncontrollable natural energy resources can not guarantee a constant amount of energy which may put reliable communications at risk. To overcome this limitation, EH from man-made radio-frequency (RF) signals has recently been proposed since such signals not only can carry information but can also serve as a convenient energy source; this is referred to as wireless power transfer.

Khaled M. Rabie and Bamidele Adebisi are with the school of Electrical Engineering, Manchester Metropolitan University, Manchester, M15 6BH, UK. (e-mails: k.rabie@mmu.ac.uk; b.adebisi@mmu.ac.uk,).

Mohamed-Slim Alouini is with King Abdullah University of Science and Technology (KAUST), Thuwal, Mekkah Province, Saudi Arabia. (e-mail: slim.alouini@kaust.edu.sa).
This concept has particularly generated considerable research interest in the so-called simultaneous wireless information and power transfer (SWIPT) networks. Although many studies have analyzed the performance of point-to-point SWIPT based systems [1]-[5], cooperative relaying SWIPT networks, where an intermediate relaying node is used to forward the source's information to the intended destination, have been by far more extensively investigated in the literature, see e.g., [6]-[8] and the reference therein. More specifically, the authors in [7] examined the performance of a half-duplex (HD) amplify-and-forward (AF) relaying network with EH where a greedy switching policy was deployed. Later on, the authors of [8] evaluated the performance of a one-way AF relaying system with three different EH protocols, namely, time-switching relaying (TSR), power-splitting relaying (PSR) and ideal relaying receiver (IRR). Furthermore, [9] considered the outage probability and ergodic capacity analysis of a twoway EH relay network. Decode-and-forward (DF) relaying with TSR and PSR EH was studied in [10] where the authors derived exact analytical expressions for the achievable throughput and ergodic capacity. Other works studying DF EH systems have also appeared in [11]-[14]. The performance of energy-constrained multiple-relay networks with relay selection is examined in [15]. Physical later security (PLS) HD EHbased multi-antenna AF relay networks was studied in [16]. In this work, the authors exploited the artificial noise signal, which is traditionally used to improve the secrecy rate, to assist in powering the energy-constrained relay. Similar works combining PLS and EH have been reported in [17]-[19].

The aforementioned relaying SWIPT systems have been limited to HD relaying costing 50\% loss in spectral efficiency. Therefore, full-duplex (FD) relaying mechanism, which exploits the scarce frequency spectrum more efficiently by supporting simultaneous signal transmission and reception over the same frequency band [20]-[22], has recently been implemented in SWIPT networks, see e.g., [23]-[28].

All the existing works on SWIPT, including the ones above, have been limited to a restricted number of fading models such as Rayleigh, Nakagami-m and Rician, which are valid to model the outdoor wireless channel. On the other hand, the analysis of SWIPT systems over indoor log-normal fading channels is scarce; in fact, to the authors' best knowledge, only one study has recently appeared in the literature investigating the performance of a HD SWIPT network with AF relaying over the log-normal fading channel [29]. It is worth noting that log-normal fading is usually used to study the communication performance in many reference scenarios. For instance, it can accurately characterize shadowing from obstacles and moving human bodies in indoor environments, and log-normal 
distribution offers a better fit for modeling fading fluctuations in indoor wireless channels [30]-[34]; it is also used to model small-scale fading for indoor ultra-wideband (UWB) communications [35], [36]. Furthermore, empirical fading channel measurements have shown that short-term and longterm fading effects over the slowly-varying indoor channel tend to get mixed and log-normal statistics become dominate; hence, it describes the distribution of the channel path gain [33], [37], [38]. RF signals in indoor wireless channels may strongly attenuate due to obstacles such as object mobility and building walls which necessitates the use of relays [39], [40].

Motivated by the above considerations, this paper is therefore dedicated to study the performance of relaying SWIPT systems over indoor log-normal channels with $\mathrm{HD}$ and FD; both $\mathrm{AF}$ and $\mathrm{DF}$ relaying schemes are adopted along with TSR, PSR and IRR EH protocols. The system performance is evaluated in terms of the ergodic outage probability ${ }^{1}$.

Therefore, the contribution of this paper is as follows. First, we derive analytical expressions of the ergodic outage probability for a dual-hop HD SWIPT system in log-normal fading with both DF and AF relaying. In this respect, TSR, PSR and IRR EH protocols are investigated for each case; hence, six distinct system configurations are studied resulting in six different analytical expressions. The second part of this work deals with FD SWIPT in log-normal fading for both DF and $\mathrm{AF}$ relaying. The other contribution resides in examining the impact of log-normal fading parameters on the ergodic outage probability as well as comparing the performance of $\mathrm{DF}$ and AF relaying in various EH protocols. Furthermore, the optimization problem of the EH time factor and powersplitting factor in the TSR and PSR schemes is addressed. Results show that when the processing energy cost of the DF relay is ignored, DF-based systems always offer better performance compared to that of $\mathrm{AF}$ relaying. It is also demonstrated that increasing the log-normal fading channel variance leads to performance degradation in all systems under study. In addition, the FD systems tend to outperform the HD ones given that the loop-back interference, caused by FD relaying, is relatively small.

The following notations are used in this paper. $f_{X}(\cdot)$, $F_{X}(\cdot)$ and $\bar{F}_{X}(\cdot)$ denote the probability density function (PDF), the cumulative distribution function (CDF) and the complementary CDF (CCDF) of the random variable (RV) $X$, respectively. $E\{\cdot\}$ and $\min \{\cdot\}$ denote the expectation operator and the minimum argument, respectively.

The rest of this paper is organized as follows. Section II briefly describes the HD and FD system models. Section III analyzes the ergodic outage probability performance of the dual-hop HD SWIPT network with DF and AF relaying, and TSR, PSR and IRR EH protocols. Section IV is dedicated to study FD with DF and AF relaying over log-normal fading channels. Numerical examples and simulation results are presented and discussed in Section V. Finally, conclusions are drawn in Section VI.

${ }^{1}$ Note that part of this paper was presented at the IEEE (GLOBECOM 2016) [41].

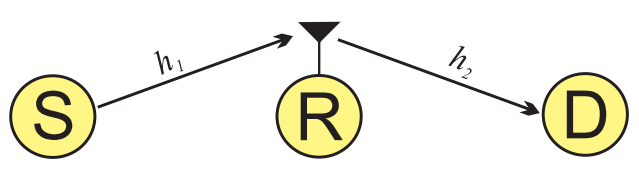

(a) HD relaying.

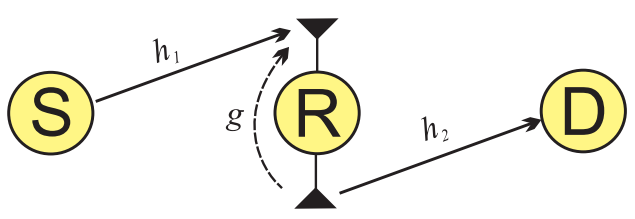

(b) FD relaying.

Figure 1: Basic bock diagram of the HD and FD relaying systems under consideration.

\section{SySTEM MOdEL}

Fig. 1 illustrates the basic system diagrams of the considered dual-hop HD and FD systems which consist of a source node, relay node and destination node. The source first transmits its data, with power $P_{s}$, to the destination via an intermediate energy-constrained relay. The relay can be either based on DF or AF. It is assumed that there is no direct link between the end nodes and that the relay is powered entirely from harvesting the energy signal transmitted by the source node. The sourceto-relay and relay-to-destination channel coefficients are denoted by $h_{1}$ and $h_{2}$ with $d_{1}$ and $d_{2}$ being the corresponding distances, respectively. Although the power consumed by the circuitry to process data at the relay is neglected in our derivations, this will be discussed in depth in the results section.

In the HD scenario, see Fig. 1(a), the relay has a singleantenna and hence the source-to-destination information transmission is accomplished over two phases. On the other hand, in FD relaying, Fig. 1(b), the relay is equipped with two antennas which allows simultaneous information reception and transmission at the relay with a loop-back interference channel denoted as $(g)$. It is important to mention that real channels are considered throughout. Note that in both HD and FD cases, the source and destination nodes are equipped with a singleantenna each; this configuration has been adopted in several studies dealing with log-normal fading channels [35], [42], [43]. In FD relaying, one antenna is dedicated to harvesting energy, and is only used for this purpose. This configuration is chosen not only for its relative ease of implementation but also because, according to [44], it attains comparable performance to the case when the two antennas are exploited for $\mathrm{EH}$.

We assume that $h_{1}^{2}$ and $h_{2}^{2}$ are independent and identically distributed log-normal RVs with parameters $L N\left(2 \mu_{h_{1}}, 4 \sigma_{h_{1}}^{2}\right)$ and $L N\left(2 \mu_{h_{2}}, 4 \sigma_{h_{2}}^{2}\right)$, respectively, where $\mu_{h_{i}}$ and $\sigma_{h_{i}}$ (both in decibels) are respectively the mean and the standard deviation of $10 \log _{10}\left(h_{i}\right), i \in\{1,2\}$. In addition, the loop-back interference channel $g^{2}$ is assumed log-normally distributed with parameters $L N\left(2 \mu_{g}, 4 \sigma_{g}^{2}\right)$; note that this is a key parameter determining the strength of the loop-back interference and hence the overall performance of FD relaying. 
As mentioned in the introduction, the system performance is evaluated in terms of the ergodic outage probability. This probability is defined as the probability that the instantaneous capacity falls below a certain threshold value $\left(C_{t h}\right)$ and can be calculated for the AF and DF relaying systems, respectively, as

$$
\mathcal{O}\left(C_{t h}\right)=\operatorname{Pr}\left\{C_{d}\left(\gamma_{d}\right)<C_{t h}\right\},
$$

and

$$
\mathcal{O}\left(C_{t h}\right)=\operatorname{Pr}\left\{\min \left\{C_{r}\left(\gamma_{r}\right), C_{d}\left(\gamma_{d}\right)\right\}<C_{t h}\right\},
$$

where $C_{r}$ and $C_{d}$ are the instantaneous capacities at the relay and destination nodes, respectively, while $\gamma_{r}$ and $\gamma_{d}$ denote the corresponding signal-to-noise ratios (SNR).

The received signal at the relay during the $\mathrm{EH}$ phase in both HD and FD can be expressed as

$$
y_{r}(t)=\sqrt{\frac{P_{s}}{d_{1}^{m}}} h_{1} s(t)+n_{a}(t),
$$

where $m$ is the path loss exponent, $s(t)$ is the information signal normalized as $\mathbb{E}\left[|s|^{2}\right]=1$ and $n_{a}(t)$ is narrowband Gaussian noise introduced by the receiving antenna at the relay with variance $\sigma_{a}^{2}$. We next derive analytical expressions of the ergodic outage probability for the systems under study.

\section{Half-Duplex Relaying System}

This section analyzes the performance of HD relaying over log-normal channels with both DF and AF relaying.

\section{A. Half-Duplex with DF Relaying}

Below, we derive analytical expressions for the HD-DF system with TSR, PSR and IRR EH protocols.

1) HD-DF-TSR System : In the TSR protocol, the time required to transmit one block from the source to the destination, also referred to as the time frame $(T)$, is divided into three time slots as shown in Fig. 2. The first time period is the EH time, $\tau T$, during which the relay harvests the power signal broadcast by the source node, where $0 \leq \tau \leq 1$ is the EH time factor. The remaining time is divided into two equal time slots used for source-to-relay and relay-to-destination information transmission.

Therefore, using (3), the harvested energy at the relay for this system can be written as

$$
E_{H}=\frac{\eta \tau T P_{s} h_{1}^{2}}{d_{1}^{m}}
$$

where $0<\eta<1$ is the EH efficiency determined mainly by the circuitry. Now, the received signal at the destination node can be expressed as

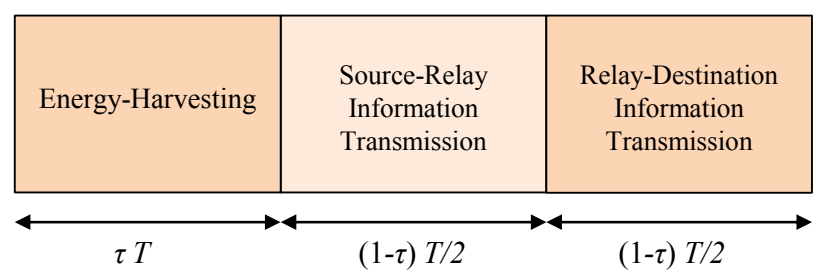

Figure 2: Time frame structure in the TSR protocol.

$$
y_{d}(t)=\sqrt{\frac{P_{r}}{d_{2}^{m}}} h_{2} \bar{s}(t)+n_{d}(t),
$$

where $\bar{s}(t)$ is the decoded version of the source signal, $n_{d}(t)=n_{a}(t)+n_{c}(t)$ is the overall noise at the destination node with variance $\sigma_{d}^{2}, n_{c}(t)$ is the noise added by the information receiver, and $P_{r}$ is the relay transmit power which is related to the harvested energy as

$$
P_{r}=\frac{E_{H}}{(1-\tau) T / 2}=\frac{2 \eta P_{s} h_{1}^{2} \tau}{(1-\tau) d_{1}^{m}} .
$$

Substituting (6) into (5) yields

$$
y_{d}(t)=\sqrt{\frac{2 \eta \tau P_{s}}{(1-\tau) d_{1}^{m} d_{2}^{m}}} h_{1} h_{2} \bar{s}(t)+n_{d}(t) .
$$

Grouping the information and noise terms in (3) and (7), we obtain the SNRs at the relay and destination nodes, respectively, as follows

$$
\begin{gathered}
\gamma_{r}=\frac{P_{s} h_{1}^{2}}{d_{1}^{m} \sigma_{r}^{2}}, \\
\gamma_{d}=\frac{2 \eta \tau P_{s} h_{1}^{2} h_{2}^{2}}{(1-\tau) d_{1}^{m} d_{2}^{m} \sigma_{d}^{2}} .
\end{gathered}
$$

Since in the TSR protocol information transmission takes place only during the time fraction $(1-\tau)$, the instantaneous capacity of the first and second links can be given by

$$
C_{i}^{H D-T S R}=\frac{(1-\tau)}{2} \log _{2}\left(1+\gamma_{i}\right)
$$

where $i \in\{r, d\}$ and the factor $\frac{1}{2}$ is a result of HD relaying.

To derive the ergodic outage probability for the HD-DFTSR system, we first write (2) as

$$
\begin{aligned}
\mathcal{O}^{T S R}\left(C_{t h}\right)= & \operatorname{Pr}\left\{\min \left\{C_{r}^{T S R}, C_{d}^{T S R}\right\}<C_{t h}\right\} \\
=1- & \operatorname{Pr}\left\{\min \left\{C_{r}^{T S R}, C_{d}^{T S R}\right\} \geq C_{t h}\right\} \\
=1- & \operatorname{Pr}\left\{C_{r}^{T S R} \geq C_{t h}, C_{d}^{T S R} \geq C_{t h}\right\} \\
=1- & \underbrace{\operatorname{Pr}\left\{C_{r}^{T S R} \geq C_{t h}\right\}}_{\mathcal{O}_{1}^{T S R}\left(C_{t h}\right)} \\
& +\underbrace{\operatorname{Pr}\left\{C_{r}^{T S R} \geq C_{t h}, C_{d}^{T S R}<C_{t h}\right\}}_{\mathcal{O}_{2}^{T S R}\left(C_{t h}\right)} .
\end{aligned}
$$


It is clear that the ergodic outage probability requires calculating two probabilities. Using (8) and (10), and substituting $X=h_{1}^{2}$, the first probability in (11) can be calculated as

$$
\begin{aligned}
\mathcal{O}_{1}^{T S R}\left(C_{t h}\right) & =\operatorname{Pr}\left\{C_{r}^{T S R} \geq C_{t h}\right\} \\
& =\operatorname{Pr}\left\{\frac{(1-\tau)}{2} \log _{2}\left(1+\frac{P_{s} X}{d_{1}^{m} \sigma_{r}^{2}}\right) \geq C_{t h}\right\} \\
& =\operatorname{Pr}\left\{\frac{P_{s} X}{d_{1}^{m} \sigma_{r}^{2}} \geq v\right\} \\
& =\operatorname{Pr}\left\{X \geq a_{1} v\right\} \\
& =1-F_{X}\left(a_{1} v\right)
\end{aligned}
$$

where $v=2^{\frac{2 C_{t h}}{1-\tau}}-1, a_{1}=d_{1}^{m} \sigma_{r}^{2} / P_{s}$ and $F_{X}(\cdot)$ denotes the $\mathrm{CDF}$ of the RV $X$. Since $X$ is log-normally distributed, its $\mathrm{CDF}$ is given by

$$
F_{X}\left(a_{1} v\right)=1-Q\left(\frac{\xi \ln \left(a_{1} v\right)-2 \mu_{h_{2}}}{2 \sigma_{h_{2}}}\right),
$$

where $\xi=10 / \ln (10)$ is a scaling constant and $Q(\cdot)$ is the Gaussian $Q$-function, given by

$$
Q(x)=\int_{x}^{\infty} \frac{1}{\sqrt{2 \pi}} \exp \left(-\frac{t^{2}}{2}\right) \mathrm{d} t .
$$

Now, using (8)-(10), and substituting $Y=h_{2}^{2}$, the second probability in (11) can be determined as

$$
\begin{aligned}
\mathcal{O}_{2}^{T S R}\left(C_{t h}\right) & =\operatorname{Pr}\left\{C_{r}^{T S R} \geq C_{t h}, C_{d}^{T S R}<C_{t h}\right\} \\
& =\operatorname{Pr}\left\{X \geq a_{1} v, \frac{2 \eta \tau P_{s} X Y}{(1-\tau) d_{1}^{m} d_{2}^{m} \sigma_{d}^{2}}<v\right\} \\
& =\operatorname{Pr}\left\{X \geq a_{1} v, Y<\frac{a_{2} v}{X}\right\},
\end{aligned}
$$

where $a_{2}=(1-\tau) d_{1}^{m} d_{2}^{m} \sigma_{d}^{2} / 2 \eta \tau P_{s}$.

Using the PDF and CDF of the log-normally distributed RVs $X$ and $Y$, we can calculate the second probability in (15) as

$$
\mathcal{O}_{2}^{T S R}\left(C_{t h}\right)=\int_{a_{1} v}^{\infty} f_{X}(z) F_{Y}\left(\frac{a_{2} v}{z}\right) \mathrm{d} z,
$$

where

$$
f_{X}(z)=\frac{\xi}{z \sqrt{8 \pi \sigma_{h_{1}}^{2}}} \exp \left(-\frac{\left(\xi \ln (z)-2 \mu_{h_{1}}\right)^{2}}{8 \sigma_{h_{1}}^{2}}\right)
$$

and

$$
F_{Y}\left(\frac{a_{2} v}{z}\right)=1-\mathrm{Q}\left(\frac{\xi \ln \left(\frac{a_{2} v}{z}\right)-2 \mu_{h_{2}}}{2 \sigma_{h_{2}}}\right) .
$$

Finally, substituting (12) and (16) into (11) yields the ergodic outage probability of the HD-DF-TSR system, given by

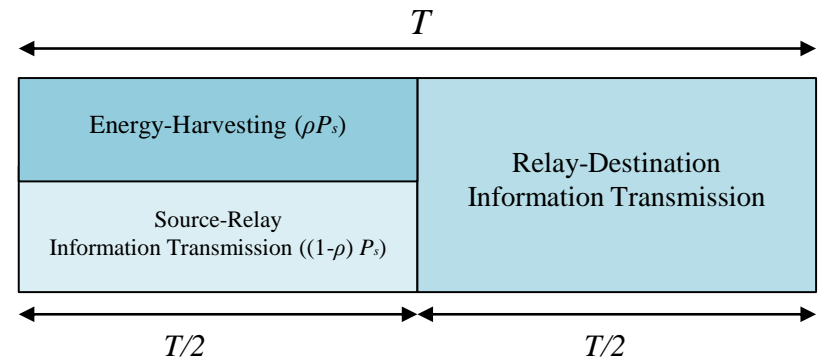

Figure 3: Time frame structure of the PSR protocol.

$$
\begin{aligned}
\mathcal{O}_{T S R}\left(C_{t h}\right)=1 & -\mathrm{Q}\left(\frac{\xi \ln \left(a_{1} v\right)-2 \mu_{h_{2}}}{2 \sigma_{h_{2}}}\right)+\frac{\xi}{\sqrt{8 \pi \sigma_{h_{1}}^{2}}} \\
& \times \int_{a_{1} v}^{\infty} \frac{1}{z} \exp \left(-\frac{\left(\xi \ln (z)-2 \mu_{h_{1}}\right)^{2}}{8 \sigma_{h_{1}}^{2}}\right) \\
& \times\left(1-\mathrm{Q}\left(\frac{\xi \ln \left(\frac{a_{2} v}{z}\right)-2 \mu_{h_{2}}}{2 \sigma_{h_{2}}}\right)\right) \mathrm{d} z .
\end{aligned}
$$

2) HD-DF-PSR System: In the PSR protocol, the block time, $T$, is divided evenly for the source-to-relay and relay-todestination transmissions as illustrated in Fig. 3. During the first half, the relay allocates a portion of the received signal power, $\rho P$, to the energy-harvester whereas the remaining power, $(1-\rho) P$, is used for information transmission, where $0 \leq \rho \leq 1$ is the power-splitting factor. Therefore, in the first time slot the received signal at the input of the energyharvester is expressed as

$$
\sqrt{\rho} y_{r}(t)=\sqrt{\frac{\rho P_{s}}{d_{1}^{m}}} h_{1} s(t)+\sqrt{\rho} n_{a}(t) .
$$

Using (20), the harvested energy at the relay node can be simply written as

$$
E_{H}=\frac{\eta \rho P_{s} h_{1}^{2} T}{2 d_{1}^{m}} .
$$

On the other hand, the base-band signal at the information receiver, $\sqrt{1-\rho} y_{r}(t)$, is given by

$$
\sqrt{1-\rho} y_{r}(t)=\sqrt{\frac{(1-\rho) P_{s}}{d_{1}^{m}}} h_{2} s(t)+n_{r}(t),
$$

where $n_{r}(t)=\sqrt{1-\rho} n_{a}(t)+n_{c}(t)$ is the overall noise at the relay with variance $\sigma_{r}^{2}=\sqrt{1-\rho} \sigma_{a}^{2}+\sigma_{c}^{2}$.

In the second time slot, the relay decodes the signal in (22), re-modulates and forwards it using the harvested energy in (21). Therefore, the received signal at the destination node can be expressed as

$$
y_{d}(t)=\sqrt{\frac{P_{r}}{d_{2}^{m}}} h_{2} \bar{s}(t)+n_{d}(t),
$$


where $P_{r}$ is the relay transmit power which is related to the harvested energy as

$$
P_{r}=\frac{E_{H}}{T / 2}=\frac{\eta \rho P_{s} h_{1}^{2}}{d_{1}^{m}} .
$$

Now, substituting (24) into (23) produces

$$
y_{d}(t)=\sqrt{\frac{\eta \rho P_{s}}{d_{1}^{m} d_{2}^{m}}} h_{1} h_{2} \bar{s}(t)+n_{d}(t) .
$$

Using (22) and (25), the SNRs at the relay and destination nodes can respectively be expressed as

$$
\begin{gathered}
\gamma_{r}=\frac{(1-\rho) P_{s} h_{1}^{2}}{d_{1}^{m} \sigma_{r}^{2}} \\
\gamma_{d}=\frac{\eta \rho P_{s} h_{1}^{2} h_{2}^{2}}{d_{1}^{m} d_{2}^{m} \sigma_{d}^{2}} .
\end{gathered}
$$

The instantaneous capacity at the relay and destination for the HD-DF-PSR system can be determined using

$$
C_{i}^{P S R}=\frac{1}{2} \log _{2}\left(1+\gamma_{i}\right),
$$

where $i \in\{r, d\}$.

Similar to the HD-DF-TSR system, the ergodic outage probability of the HD-DF-PSR approach can be calculated as

$$
\begin{aligned}
\mathcal{O}^{P S R}\left(C_{t h}\right)= & 1-\underbrace{\operatorname{Pr}\left\{C_{r}^{P S R} \geq C_{t h}\right\}}_{\mathcal{O}_{1}^{P S R}\left(C_{t h}\right)} \\
& +\underbrace{\operatorname{Pr}\left\{C_{r}^{P S R} \geq C_{t h}, C_{d}^{P S R}<C_{t h}\right\}}_{\mathcal{O}_{2}^{P S R}\left(C_{t h}\right)} .
\end{aligned}
$$

Using (26) and (28), the first probability in (29) can be written as

$$
\begin{aligned}
\mathcal{O}_{1}^{P S R}\left(C_{t h}\right) & =\operatorname{Pr}\left\{C_{r}^{P S R} \geq C_{t h}\right\} \\
& =\operatorname{Pr}\left\{\frac{1}{2} \log _{2}\left(1+\frac{(1-\rho) P_{s} X}{d_{1}^{m} \sigma_{r}^{2}}\right) \geq C_{t h}\right\} \\
& =\operatorname{Pr}\left\{\frac{(1-\rho) P_{s} X}{d_{1}^{m} \sigma_{r}^{2}} \geq R\right\} \\
& =\operatorname{Pr}\left\{X \geq b_{1} R\right\} \\
& =1-F_{X}\left(b_{1} R\right)
\end{aligned}
$$

where $R=2^{2 C_{t h}}-1, b_{1}=d_{1}^{m} \sigma_{r}^{2} /(1-\rho) P_{s}$ and $F_{X}(\cdot)$ represents the $\mathrm{CDF}$ of $X$ which is given in this case as

$$
F_{Y}\left(b_{1} R\right)=1-\mathrm{Q}\left(\frac{\xi \ln \left(b_{1} R\right)-2 \mu_{h_{2}}}{2 \sigma_{h_{2}}}\right) .
$$

Using (26)-(28), the second probability in (29) can be calculated as follows

$$
\begin{aligned}
\mathcal{O}_{2}^{P S R}\left(C_{t h}\right) & =\operatorname{Pr}\left\{C_{r}^{P S R} \geq C_{t h}, C_{d}^{P S R}<C_{t h}\right\} \\
& =\operatorname{Pr}\left\{X \geq b_{1} R, \frac{\eta \rho P_{s} X Y}{d_{1}^{m} d_{2}^{m} \sigma_{d}^{2}}<R\right\} \\
& =\operatorname{Pr}\left\{X \geq b_{1} R, Y<\frac{b_{2} R}{X}\right\}
\end{aligned}
$$

where $b_{2}=d_{1}^{m} d_{2}^{m} \sigma_{d}^{2} / \eta \rho P_{s}$.

Now, using the PDF and CDF of the RVs $X$ and $Y$, we can express $\mathcal{O}_{2}^{P S R}\left(C_{t h}\right)$ as

$$
\mathcal{O}_{2}^{P S R}\left(C_{t h}\right)=\int_{b_{1} R}^{\infty} f_{X}(z) F_{Y}\left(\frac{b_{2} R}{z}\right) \mathrm{d} z
$$

where

$$
f_{X}(z)=\frac{\xi}{z \sqrt{8 \pi \sigma_{h_{1}}^{2}}} \exp \left(-\frac{\left(\xi \ln (z)-2 \mu_{h_{1}}\right)^{2}}{8 \sigma_{h_{1}}^{2}}\right)
$$

and

$$
F_{Y}\left(\frac{b_{2} R}{z}\right)=1-\mathrm{Q}\left(\frac{\xi \ln \left(\frac{b_{2} R}{z}\right)-2 \mu_{h_{2}}}{2 \sigma_{h_{2}}}\right) .
$$

Finally, substituting (30) and (33) into (29) produces the ergodic outage probability of the HD-DF-PSR system in lognormal fading, expressed as

$$
\begin{aligned}
\mathcal{O}^{P S R}\left(C_{t h}\right)= & -\mathrm{Q}\left(\frac{\xi \ln \left(b_{1} R\right)-2 \mu_{h_{2}}}{2 \sigma_{h_{2}}}\right)+\frac{\xi}{\sqrt{8 \pi \sigma_{h_{1}}^{2}}} \\
& \times \int_{b_{1} R}^{\infty} \frac{1}{z} \exp \left(-\frac{\left(\xi \ln (z)-2 \mu_{h_{1}}\right)^{2}}{8 \sigma_{h_{1}}^{2}}\right) \\
& \times\left(1-\mathrm{Q}\left(\frac{\xi \ln \left(\frac{b_{2} R}{z}\right)-2 \mu_{h_{2}}}{2 \sigma_{h_{2}}}\right)\right) \mathrm{d} z .
\end{aligned}
$$

3) HD-DF-IRR System: Unlike the TSR and PSR protocols, the IRR scheme is capable of concurrently processing information and harvesting energy from the same received signal. Therefore, the signal received at the information receiver of the relay can be expressed as

$$
y_{r}(t)=\sqrt{\frac{P_{s}}{d_{1}^{m}}} h_{1} s(t)+n_{r}(t),
$$

and the harvested energy and the relay transmit power can therefore be given respectively as

$$
\begin{gathered}
E_{H}=\frac{\eta P_{s} h_{1}^{2}}{d_{1}^{m}}(T / 2), \\
P_{r}=\frac{2 E_{H}}{T}=\frac{\eta P_{s} h_{1}^{2}}{d_{1}^{m}} .
\end{gathered}
$$

Using (39), the received signal at the destination can be written as 


$$
y_{d}(t)=\sqrt{\frac{\eta P_{s}}{d_{1}^{m} d_{2}^{m}}} h_{1} h_{2} \bar{s}(t)+n_{d}(t) .
$$

Now, using (37) and (40), the SNRs at the relay and destination nodes in the HD-DF-IRR system can be respectively expressed as

$$
\gamma_{r}=\frac{P_{s} h_{1}^{2}}{d_{1}^{m} \sigma_{r}^{2}}
$$

and

$$
\gamma_{d}=\frac{\eta P_{s} h_{1}^{2} h_{2}^{2}}{d_{1}^{m} d_{2}^{m} \sigma_{d}^{2}}
$$

The ergodic outage probability of the HD-DF-TSR system can be calculated as

$$
\begin{aligned}
\mathcal{O}^{I R R}\left(C_{t h}\right) & =1-\underbrace{\operatorname{Pr}\left\{C_{r}^{I R R} \geq C_{t h}\right\}}_{\mathcal{O}_{1}^{I R R}\left(C_{t h}\right)} \\
& +\underbrace{\operatorname{Pr}\left\{C_{r}^{I R R} \geq C_{t h}, C_{d}^{I R R}<C_{t h}\right\}}_{\mathcal{O}_{2}^{I R R}\left(C_{t h}\right)} .
\end{aligned}
$$

The first probability $\mathcal{O}_{1}^{I R R}\left(C_{t h}\right)$ can be given by

$$
\begin{aligned}
\mathcal{O}_{1}^{I R R}\left(C_{t h}\right) & =\operatorname{Pr}\left\{C_{r}^{I R R} \geq C_{t h}\right\} \\
& =\operatorname{Pr}\left\{\frac{1}{2} \log _{2}\left(1+\frac{P_{s} X}{d_{1}^{m} \sigma_{r}^{2}}\right) \geq C_{t h}\right\} \\
& =\operatorname{Pr}\left\{\frac{P_{s} X}{d_{1}^{m} \sigma_{r}^{2}} \geq R\right\} \\
& =\operatorname{Pr}\left\{X \geq c_{1} R\right\} \\
& =1-F_{X}\left(c_{1} R\right),
\end{aligned}
$$

where $c_{1}=d_{1}^{m} \sigma_{r}^{2} / P_{s}$ and $F_{X}(\cdot)$ represents the CDF of $X$ expressed in this case as

$$
F_{Y}\left(c_{1} R\right)=1-\mathrm{Q}\left(\frac{\xi \ln \left(c_{1} R\right)-2 \mu_{h_{2}}}{2 \sigma_{h_{2}}}\right) .
$$

Using (28), (41) and (42), the second probability in (43) can be written as

$$
\begin{aligned}
\mathcal{O}_{2}^{I R R}\left(C_{t h}\right) & =\operatorname{Pr}\left\{C_{r}^{I R R} \geq C_{t h}, C_{d}^{I R R}<C_{t h}\right\} \\
& =\operatorname{Pr}\left\{X \geq c_{1} R, \frac{\eta P_{s} X Y}{d_{1}^{m} d_{2}^{m} \sigma_{d}^{2}}<R\right\} \\
& =\operatorname{Pr}\left\{X \geq c_{1} R, Y<\frac{c_{2} R}{X}\right\},
\end{aligned}
$$

where $c_{2}=d_{1}^{m} d_{2}^{m} \sigma_{d}^{2} / \eta P_{s}$.

Using the PDF and CDF of the RVs $X$ and $Y$, we get

$$
\mathcal{O}_{2}^{I R R}\left(C_{t h}\right)=\int_{c_{1} R}^{\infty} f_{X}(z) F_{Y}\left(\frac{c_{2} R}{z}\right) \mathrm{d} z
$$

where

$$
f_{X}(z)=\frac{\xi}{z \sqrt{8 \pi \sigma_{h_{1}}^{2}}} \exp \left(-\frac{\left(\xi \ln (z)-2 \mu_{h_{1}}\right)^{2}}{8 \sigma_{h_{1}}^{2}}\right)
$$

and

$$
F_{Y}\left(\frac{c_{2} R}{z}\right)=1-\mathrm{Q}\left(\frac{\xi \ln \left(\frac{c_{2} R}{z}\right)-2 \mu_{h_{2}}}{2 \sigma_{h_{2}}}\right) .
$$

Finally, substituting (44) and (47) into (43) we obtain the ergodic outage probability of the HD-DF-IRR system as

$$
\begin{aligned}
\mathcal{O}^{I R R}\left(C_{t h}\right)=1 & -\mathrm{Q}\left(\frac{\xi \ln \left(c_{1} R\right)-2 \mu_{h_{2}}}{2 \sigma_{h_{2}}}\right)+\frac{\xi}{\sqrt{8 \pi \sigma_{h_{1}}^{2}}} \\
& \times \int_{c_{1} R}^{\infty} \frac{1}{z} \exp \left(-\frac{\left(\xi \ln (z)-2 \mu_{h_{1}}\right)^{2}}{8 \sigma_{h_{1}}^{2}}\right) \\
& \times\left(1-\mathrm{Q}\left(\frac{\xi \ln \left(\frac{c_{2} R}{z}\right)-2 \mu_{h_{2}}}{2 \sigma_{h_{2}}}\right)\right) \mathrm{d} z .
\end{aligned}
$$

\section{B. Half-Duplex with AF Relaying}

In this section, we analyze the ergodic outage probability of the HD system with AF relaying for different $\mathrm{EH}$ protocols. Although some of the results below have been derived in [29], we feel that presenting them here is important to make the current work self-contained.

1) HD-AF-TSR System: In this system, the signal received at the relay during the EH time is given by (3), and therefore the harvested energy is equal to (4). After the baseband processing and amplification at the relay, the relay transmit signal can be written as

$$
r(t)=\sqrt{\frac{P_{s} P_{r}}{d_{1}^{m}}} G h_{1} s(t)+\sqrt{P_{r}} G n_{r}(t),
$$

where $P_{r}$ is given by (6), $n_{r}(t)=n_{a}(t)+n_{c}(t)$ with variance $\sigma_{r}^{2}=\sigma_{a}^{2}+\sigma_{c}^{2}$ and $G$ is the relay gain given by $G=\frac{1}{\sqrt{\frac{P_{s}^{s}}{d_{1}^{2}} h_{1}^{2}+\sigma_{r}^{2}}}$. The received signal at the destination can then be expressed as

$$
y_{d}(t)=\sqrt{\frac{P_{s} P_{r}}{d_{1}^{m} d_{2}^{m}}} G h_{1} h_{2} s(t)+\sqrt{\frac{P_{r}}{d_{2}^{m}}} G h_{2} n_{r}(t)+n_{d}(t) .
$$

Using (6) and (52), along with some basic algebraic manipulations, the SNR at the destination can be obtained as

$$
\gamma_{d}=\frac{2 \eta \tau P_{s} h_{1}^{2} h_{2}^{2}}{2 \eta \tau d_{1}^{m} \sigma_{r}^{2} h_{2}^{2}+(1-\tau) d_{1}^{m} d_{2}^{m} \sigma_{d}^{2}},
$$

which can also be simplified to

$$
\gamma_{d}=\frac{a_{1} h_{1}^{2} h_{2}^{2}}{a_{3} h_{2}^{2}+a_{2}}
$$


where $a_{1}=2 \eta \tau P_{s}, \quad a_{2}=2 \eta \tau d_{1}^{m} \sigma_{r}^{2}$ and $a_{3}=$ $(1-\tau) d_{1}^{m} d_{2}^{m} \sigma_{d}^{2}$.

Now, substituting (54) into (1), we can determine the ergodic outage probability for the HD-AF-TSR system as

$$
\begin{aligned}
\mathcal{O}^{T S R}\left(C_{t h}\right) & =\operatorname{Pr}\left\{\frac{a_{1} X Y}{a_{3} Y+a_{2}}<v\right\} \\
& =\operatorname{Pr}\left\{Y<\frac{a_{2} v}{a_{1} X-a_{3} v}\right\} .
\end{aligned}
$$

Since $Y$ is always a positive value, we can express the probability above as

$$
\mathcal{O}^{T S R}\left(C_{t h}\right)= \begin{cases}\operatorname{Pr}\left(Y<\frac{v a_{2}}{a_{1} X-\nu a_{3}}\right), & X<\frac{v a_{3}}{a_{1}} \\ \operatorname{Pr}\left(Y>\frac{v a_{2}}{a_{1} X-v a_{3}}\right)=1, & X>\frac{v a_{3}}{a_{1}} .\end{cases}
$$

The ergodic outage probability can now be calculated as

$$
\begin{aligned}
\mathcal{O}^{T S R}\left(C_{t h}\right)= & \int_{0}^{\frac{v a_{3}}{a_{1}}} f_{X}(z) \mathrm{d} z \\
& +\int_{\frac{v a_{3}}{a_{1}}}^{\infty} f_{X}(z) \underbrace{\operatorname{Pr}\left(Y \leq \frac{v a_{2}}{a_{1} z-v a_{3}}\right)}_{F_{Y}(\cdot)} \mathrm{d}(55)
\end{aligned}
$$

where $f_{X}(\cdot)$ is the PDF of $X$ and the probability in the second integral represents the $\mathrm{CDF}$ of $Y$, i.e., $F_{Y}(\cdot)$; these $\mathrm{PDF}$ and $\mathrm{CDF}$ can be given respectively as

$$
f_{X}(z)=\frac{\xi}{z \sqrt{8 \pi \sigma_{h_{1}}^{2}}} \exp \left(-\frac{\left(\xi \ln (z)-2 \mu_{h_{1}}-\xi \ln \left(a_{1}\right)\right)^{2}}{8 \sigma_{h_{1}}^{2}}\right)
$$

and

$$
F_{Y}(\Gamma)=1-\mathrm{Q}\left(\frac{\xi \ln (\Gamma)-2 \mu_{h_{2}}}{2 \sigma_{h_{2}}}\right)
$$

where $\Gamma=\frac{v a_{3}}{z-v a_{2}}$.

Finally, substituting (58) and (59) into (57) gives the ergodic outage probability of the HF-AF-TSR system as

$$
\begin{aligned}
\mathcal{O}^{T S R}\left(C_{t h}\right) & =1-\frac{\xi}{\sqrt{8 \pi \sigma_{h_{1}}^{2}}} \int_{v a_{2}}^{\infty} \frac{1}{z} \mathrm{Q}\left(\frac{\xi \ln (\Gamma)-2 \mu_{h_{2}}}{2 \sigma_{h_{2}}}\right) \\
& \times \exp \left(-\frac{\left(\xi \ln (z)-\left(2 \mu_{h_{1}}+\xi \ln \left(a_{1}\right)\right)\right)^{2}}{8 \sigma_{h_{1}}^{2}}\right) \mathrm{d} z .
\end{aligned}
$$

2) HD-AF-PSR System: In this system, the received signal at the input of the energy-harvester can also be given by (20), and therefore the harvested energy is equal to (21). In light of this, the relay transmit signal can be expressed as

$$
r(t)=\sqrt{\frac{(1-\rho) P_{s} P_{r}}{d_{1}^{m}}} G h_{1} s(t)+\sqrt{P_{r}} G n_{r}(t),
$$

where $n_{r}(t)=\sqrt{1-\rho} n_{a}(t)+n_{c}(t), P_{r}$ is defined in (24) and $G$ is given for the HD-AF-PSR system as $G=$ $\frac{1}{\sqrt{\frac{(1-\rho) P_{s}}{d_{1}^{12}}} h_{1}^{2}+\sigma_{r}^{2}}$. We can now express the signal received at the destination node as

$$
y_{d}(t)=\sqrt{\frac{(1-\rho) P_{s} P_{r}}{d_{1}^{m} d_{2}^{m}}} G h_{1} h_{2} s(t)+\sqrt{\frac{P_{r}}{d_{2}^{m}}} G h_{2} n_{r}(t)+n_{d}(t) .
$$

Substituting (24) into (62) and with some basic algebraic manipulations, we obtain the SNR at the destination as

$$
\gamma_{d}=\frac{\eta \rho(1-\rho) P_{s} h_{1}^{2} h_{2}^{2}}{\eta \rho d_{1}^{m} \sigma_{c}^{2} h_{2}^{2}+\eta \rho(1-\rho) d_{1}^{m} \sigma_{a}^{2} h_{2}^{2}+(1-\rho) d_{1}^{m} d_{2}^{m} \sigma_{d}^{2}} .
$$

Using $b_{1}=\eta \rho(1-\rho) P_{s}, \quad b_{2}=\eta \rho d_{1}^{m} \sigma_{c}^{2}, \quad b_{3}=$ $\eta \rho(1-\rho) d_{1}^{m} \sigma_{a}^{2}$ and $b_{4}=(1-\rho) d_{1}^{m} d_{2}^{m} \sigma_{d}^{2}$, we can rewrite (63) in the following simplified form

$$
\gamma_{d}=\frac{b_{1} h_{1}^{2} h_{2}^{2}}{b_{2} h_{2}^{2}+b_{3} h_{2}^{2}+b_{4}} .
$$

Following the same procedure as in the HD-AF-TSR system, it is straightforward to show that the ergodic outage probability of the HD-AF-PSR system can be calculated as

$$
\begin{aligned}
\mathcal{O}^{P S R}\left(C_{t h}\right) & =1-\frac{\xi}{\sqrt{8 \pi \sigma_{h_{1}}^{2}}} \int_{v\left(b_{2}+b_{3}\right)}^{\infty} \frac{1}{z} \mathrm{Q}\left(\frac{\xi \ln (\Lambda)-2 \mu_{h_{2}}}{2 \sigma_{h_{2}}}\right) \\
& \times \exp \left(-\frac{\left(\xi \ln (z)-2 \mu_{h_{1}}-\xi \ln \left(b_{1}\right)\right)^{2}}{8 \sigma_{h_{1}}^{2}}\right) \mathrm{d} z,
\end{aligned}
$$

where $\Lambda=\frac{v b_{4}}{z-v b_{2}-v b_{3}}$. For more details the reader may refer to [29].

3) HD-AF-IRR System: The harvested energy and relay transmit power in this system can also be given by (38) and (39), respectively. Recalling that the relay has a gain of $G$, we can write the received signal at the destination as

$y_{d}(t)=\sqrt{\frac{P_{s} P_{r}}{d_{1}^{m} d_{2}^{m}}} G h_{1} h_{2} s(t)+\sqrt{\frac{P_{r}}{d_{2}^{m}}} G h_{2} n_{r}(t)+n_{d}(t)$, 
where $G=\frac{1}{\sqrt{\frac{P_{s}}{d^{m}} h_{1}^{2}+\sigma_{r}^{2}}}$.

Substituting (39) into (66), and then grouping the information and noise signals, the SNR at the destination node can be given by

$$
\gamma_{d}=\frac{\eta P_{s} h_{1}^{2} h_{2}^{2}}{\eta d_{1}^{m} \sigma_{r}^{2} h_{2}^{2}+d_{1}^{m} d_{2}^{m} \sigma_{d}^{2}},
$$

which can also be written, for more convenience, as

$$
\gamma_{d}=\frac{c_{1} h_{1}^{2} h_{2}^{2}}{c_{2} h_{2}^{2}+c_{3}}
$$

where $c_{1}=\eta P_{s}, c_{2}=\eta d_{1}^{m} \sigma_{r}^{2}$ and $c_{3}=d_{1}^{m} d_{2}^{m} \sigma_{d}^{2}$.

Following the same procedure as in Sec. III-B-3, we can show that the ergodic outage probability of the HD-AF-IRR system can be calculated as

$$
\begin{aligned}
\mathcal{O}^{I R R}\left(C_{t h}\right) & =1-\frac{\xi}{\sqrt{8 \pi \sigma_{h_{1}}^{2}}} \int_{v c_{2}}^{\infty} \frac{1}{z} \mathrm{Q}\left(\frac{\xi \ln (\Upsilon)-2 \mu_{h_{2}}}{2 \sigma_{h_{2}}}\right) \\
& \times \exp \left(-\frac{\left(\xi \ln (z)-2 \mu_{h_{1}}-\xi \ln \left(c_{1}\right)\right)^{2}}{8 \sigma_{h_{1}}^{2}}\right) \mathrm{d} z,
\end{aligned}
$$

where $\Upsilon=\frac{v c_{3}}{z-v c_{2}}$.

\section{Full-DupleX RELAYING SySTEM}

This section is dedicated to analyze the performance of a dual-hop FD network with DF and AF relaying based on the TSR EH protocol. Recall that unlike HD, in FD the relay is equipped with two antennas and this allows simultaneous reception and transmission of information at the relay, see Fig. $1(b)$.

To begin with, in the first time slot, the received signal at the relay can also be given by (3) and the relay transmit power will have the following form

$$
P_{r}=\frac{E_{H}}{(1-\tau) T}=\frac{\eta \tau P_{s} h_{1}^{2}}{(1-\tau) d_{1}^{m}} .
$$

In the second time slot (information transmission phase), the received signal at the relay is given by

$$
y_{r}(t)=\sqrt{\frac{P_{s}}{d_{1}^{m}}} h_{1} s(t)+g r(t)+n_{a}(t),
$$

where $g$ is the loop-back interference channel and $r(t)$ is the loop-back interference signal due to FD relaying and it satisfies the following $E\left\{|r(t)|^{2}\right\}=P_{r}$.

It should be pointed out that in the FD system, since the relay knows its own signal, it usually applies interference cancellation to reduce the loop-back interference. Therefore, we can now write the post-cancellation signal at the relay as

$$
y_{r}(t)=\sqrt{\frac{P_{s}}{d_{1}^{m}}} h_{1} s(t)+\hat{g} \hat{r}(t)+n_{a}(t),
$$

where $\hat{g}$ is the residual loop-back interference channel caused by imperfect interference cancellation and $E\left\{|\hat{r}(t)|^{2}\right\}=P_{r}$. Below, we derive the ergodic outage probability expressions for both FD-DF and FD-AF relaying systems.

\section{A. Full-Duplex with DF Relaying (FD-DF-TSR)}

When DF relaying is applied, the relay will decode and forward the source signal; hence, the received signal at the destination can be expressed as

$$
y_{d}(t)=\sqrt{\frac{P_{r}}{d_{2}^{m}}} h_{2} \bar{s}(t)+n_{d}(t) .
$$

Using (70), (72) and (73), the SNRs at the relay and destination in the FD-DF-TSR system can be written respectively as

$$
\begin{aligned}
\gamma_{r} & =\frac{P_{s} h_{1}^{2}}{P_{r} d_{1}^{m} g^{2}} \\
& =\frac{1-\tau}{\eta \tau g^{2}}
\end{aligned}
$$

and

$$
\begin{aligned}
\gamma_{d} & =\frac{P_{r} h_{2}^{2}}{d_{2}^{m} \sigma_{d}^{2}} \\
& =\frac{\eta \tau P_{s} h_{1}^{2} h_{2}^{2}}{(1-\tau) d_{1}^{m} d_{2}^{m} \sigma_{d}^{2}}
\end{aligned}
$$

The instantaneous capacity of the source-to-relay and relayto-destination links can now be expressed as

$$
C_{i}^{F D-D F}=(1-\tau) \log _{2}\left(1+\gamma_{i}\right)
$$

where $i \in\{r, d\}$. Comparing (10) and (76), it is obvious that the factor $\frac{1}{2}$ is no longer present in (76) due to the FD nature of the relay.

Now, the ergodic outage probability for this system can be determined as

$\mathcal{O}^{F D-D F}\left(C_{t h}\right)=\operatorname{Pr}\left\{\min \left\{C_{r}^{F D-D F}, C_{d}^{F D-D F}\right\}<C_{t h}\right\}$.

Using (74)-(76), and substituting $Z=h_{1}^{2} h_{2}^{2}$ and $W=g^{2}$, the probability in (77) can be expressed as

$$
\mathcal{O}^{F D-D F}\left(C_{t h}\right)=\operatorname{Pr}\left\{\min \left\{\frac{1-\tau}{\eta \tau W}, \frac{\eta \tau P_{s} Z}{(1-\tau) d_{1}^{m} d_{2}^{m} \sigma_{d}^{2}}\right\}<v\right\},
$$

where $v=2^{\frac{C_{t h}}{1-\tau}}-1$.

Using the fact that the RVs $Z$ and $W$ are independent, the ergodic outage probability of the FD-DF-TSR system can be given by 
$\mathcal{O}^{F D-D F}\left(C_{t h}\right)=1-\bar{F}_{W}\left(\frac{\eta \tau}{1-\tau} v\right) \bar{F}_{Z}\left(\frac{d_{1}^{m} d_{2}^{m} \sigma_{d}^{2}}{(1-\tau) \eta \tau P_{s}} v\right)$,

where $\bar{F}_{W}(\cdot)$ and $\bar{F}_{Z}(\cdot)$ are the CCDFs of $W$ and $Z$, respectively.

The first CCDF, $\bar{F}_{W}(\cdot)$, is straightforward to obtain since the RV $W$ is log-normally distributed. On the other hand, the $\mathrm{RV} W$ is a product of two log-normally distributed RVs. Using the properties of the log-normal distribution, it can be shown that the CCDF of $Z$ is

$$
\bar{F}_{Z}(\cdot)=\mathrm{Q}\left(\frac{\xi \ln (\Delta)-2\left(\mu_{h_{1}}+\mu_{h_{2}}\right)}{\sqrt{2}\left(\sigma_{h_{1}}+\sigma_{h_{2}}\right)}\right),
$$

where $\Delta=\frac{d_{1}^{m} d_{2}^{m} \sigma_{d}^{2}}{(1-\tau) \eta \tau P_{s}} v$.

With this in mind, we can finally express the ergodic outage probability of the FD-DF-TSR system as

$$
\begin{aligned}
\mathcal{O}^{F D-D F}\left(C_{t h}\right)=1 & -\mathrm{Q}\left(\frac{\xi \ln \left(\frac{\eta \tau}{1-\tau} v\right)+2 \mu_{g}}{2 \sigma_{g}}\right) \\
& \times \mathrm{Q}\left(\frac{\xi \ln (\Delta)-2 \sum_{i \in\{1,2\}} \mu_{h_{i}}}{\sqrt{2} \sum_{i \in\{1,2\}} \sigma_{h_{i}}}\right) .
\end{aligned}
$$

\section{B. Full-Duplex with AF Relaying (FD-AF-TSR)}

In the case of FD-AF system, the received signal at the relay (72) will be amplified and then forwarded to the destination node. Hence, the received signal at the destination can be expressed as

$$
\begin{array}{r}
y_{r}(t)=\sqrt{\frac{P_{s} P_{r}}{d_{1}^{m} d_{2}^{m}}} h_{1} h_{2} G s(t)+\sqrt{\frac{P_{r}}{d_{2}^{m}}} h_{2} g G r(t) \\
+\sqrt{\frac{P_{r}}{d_{2}^{m}}} h_{2} G n_{a}(t)+n_{d}(t),
\end{array}
$$

where $P_{r}$ is given by (70) and $G$ is the relay gain defined as

$$
G=\frac{1}{\sqrt{\frac{P_{s}}{d_{1}^{m}} h_{1}^{2}+\hat{g}^{2} P_{r}+\sigma_{r}^{2}}} .
$$

Grouping the information signal and noise terms in (82), we can write the SNR at the destination as

$$
\gamma_{d}=\frac{P_{s} h_{1}^{2} h_{2}^{2}}{d_{1}^{m} d_{2}^{m} g^{2}\left(\frac{P_{s} h_{1}^{2} \sigma_{r}^{2}}{P_{r} g^{2} d_{1}^{m}}+\frac{P_{r} h_{2}^{2}}{d_{2}^{m}}+\sigma_{r}^{2}\right)} .
$$

Note that the instantaneous capacity of the FD-AF-TSR system is determined using

$$
C_{d}^{F D-A F}=(1-\tau) \log _{2}\left(1+\gamma_{d}\right)
$$

Similar to the DF scenario, comparing (10) and (85), it can evident that the factor $\frac{1}{2}$ is no longer present (85) due to the FD relaying.

Now, using the definition in (1) along with (84) and (85), we can calculate the ergodic outage probability of the FD-AFTSR system as

$$
\begin{aligned}
\mathcal{O}^{F D-A F}\left(C_{t h}\right) & =\operatorname{Pr}\left\{C_{d}^{F D-A F}\left(\gamma_{d}\right)<C_{t h}\right\}, \\
& =\operatorname{Pr}\left\{\frac{P_{s} h_{1}^{2} h_{2}^{2}}{d_{1}^{m} d_{2}^{m} g^{2}\left(\frac{P_{s} h_{1}^{2} \sigma_{r}^{2}}{P_{r} g^{2} d_{1}^{m}}+\frac{P_{r} h_{2}^{2}}{d_{2}^{m}}+\sigma_{r}^{2}\right)}<180(0),\right.
\end{aligned}
$$

where $v=2^{\frac{C_{t h}}{1-\tau}}-1$.

Using the substitutions $k=\eta \tau /(1-\tau), Z=h_{1}^{2} h_{2}^{2}$ and $W=g^{2}$, along with some algebraic manipulations, we can rewrite (86) as

$$
\mathcal{O}^{F D-A F}\left(C_{t h}\right)=\operatorname{Pr}\left\{Z<\frac{d_{1}^{m} d_{2}^{m} v \sigma_{r}^{2}\left(\frac{1}{k}+W\right)}{P_{s}-P_{s} k v W}\right\} .
$$

We know that $Z$ is always a positive value; hence, the probability in (87) can be rewritten in the following form

$\mathcal{O}^{F D-A F}\left(C_{t h}\right)= \begin{cases}\operatorname{Pr}\left(Z \leq \frac{d_{1}^{m} d_{2}^{m} v \sigma_{r}^{2}\left(\frac{1}{k}+W\right)}{P_{s}-P_{s} k v W}\right), & W<\frac{1}{k v} \\ \operatorname{Pr}\left(Z>\frac{d_{1}^{m} d_{2}^{m} v \sigma_{r}^{2}\left(\frac{1}{k}+W\right)}{P_{s}-P_{s} k v W}\right)=1, & W>\frac{1}{k v} .\end{cases}$

This probability can be calculated as follows

$\mathcal{O}^{F D-A F}\left(C_{t h}\right)=1-\int_{0}^{\frac{1}{k v}} f_{W}(z) \bar{F}_{Z}\left(\frac{d_{1}^{m} d_{2}^{m} v \sigma_{r}^{2}\left(\frac{1}{k}+z\right)}{P_{s}-P_{s} k v z}\right) \mathrm{d} z$

where $f_{W}(\cdot)$ is the PDF of $W$ given by

$$
f_{W}(z)=\frac{\xi}{z \sqrt{8 \pi \sigma_{g}^{2}}} \exp \left(-\frac{\left(\xi \ln (z)-2 \mu_{g}\right)^{2}}{8 \sigma_{g}^{2}}\right)
$$

and $\bar{F}_{Z}(\cdot)$ is the CCDF of $Z$ defined as

$$
\bar{F}_{Z}(z)=\mathrm{Q}\left(\frac{\xi \ln \left(\frac{d_{1}^{m} d_{2}^{m} v \sigma_{r}^{2}\left(\frac{1}{k}+z\right)}{P_{s}-P_{s} k v}\right)-2 \sum_{i \in\{1,2\}} \mu_{h_{i}}}{\sqrt{2} \sum_{i \in\{1,2\}} \sigma_{h_{i}}}\right) .
$$

Finally, substituting (90) and (91) into (89), we can express the ergodic outage probability of the FD-AF-TSR system as in (92), shown at the top of the next page, where $\Gamma=$ $\frac{d_{1}^{m} d_{2}^{m} v \sigma_{r}^{2}\left(\frac{1}{k}+z\right)}{P_{s}-P_{s} k v z}$. 


$$
\mathcal{O}^{F D-A F}\left(C_{t h}\right)=1-\frac{\xi}{\sqrt{8 \pi \sigma_{h_{1}}^{2}}} \int_{0}^{\frac{1}{k v}} \frac{1}{z} \mathrm{Q}\left(\frac{\xi \ln (\Gamma)-2 \sum_{i \in\{1,2\}} \mu_{h_{i}}}{\sqrt{2} \sum_{i \in\{1,2\}} \sigma_{h_{i}}}\right) \exp \left(-\frac{\left(\xi \ln (z)-2 \mu_{h_{1}}\right)^{2}}{8 \sigma_{h_{1}}^{2}}\right) \mathrm{d} z
$$

\section{Results AND Discussions}

In this section, we present some numerical examples of the derived expressions above along with Monte Carlo simulations. Unless specified otherwise, we use in our evaluations the following system parameters: $P_{s}=1 \mathrm{~W}, \eta=1, m=2$, $d_{1}=d_{2}=5 \mathrm{~m}, C_{t h}=2 \mathrm{bps} / \mathrm{Hz}, \sigma_{1}^{2}=\sigma_{2}^{2}=4 \mathrm{~dB}$, $\mu_{1}=\mu_{2}=3 \mathrm{~dB}$ and $\sigma_{r}^{2}=\sigma_{d}^{2}=2 \sigma_{r a}^{2}=2 \sigma_{r c}^{2}=0.005 \mathrm{~W}$.

\section{A. The Impact of $\tau$ and $\rho$ on the Performance of HD-DF and HD-AF Systems}

This section discusses and compares the effect of the EH parameters $\tau$ (EH time factor in the TAR approach) and $\rho$ (power splitting factor in the PSR approach) on the ergodic outage probability of the HD-DF-TSR, HD-DF-PSR, HD-AFTSR and HD-AF-PSR systems. To achieve this, we plot in Fig. 4 the analytical and simulated ergodic outage probability for the four systems as a function of $\tau$ and $\rho$. Note that the analytical results are obtained from (19), (36), (60) and (65) for these systems, respectively. The good agreement between the analytical and simulated results clearly indicates the accuracy of our analysis. It can be seen from the results in Fig. 4 that DF relaying, in both TSR and PSR systems, tends to offer slightly better performance in comparison to that of AF relaying. This is mainly because the processing energy cost of DF relaying, which is of course higher than that of AF relaying, is ignored here; this will be investigated in more details later. In addition, it is worthwhile pointing out that this improvement is more pronounced in the TSR-based system compared to that in the PSR-based approach. Another interesting observation one can see from these results is the significant deterioration in the ergodic outage probability when $\tau$ or $\rho$ approach either zero or one due to the fact that the harvested energy becomes either too small or unnecessarily too large (leading to no resources left for data transmission). Therefore, optimizing these parameters is of great importance to maximize the system performance; this phenomena is investigated thoroughly below. It is worth mentioning that it is possible to improve the performance by allocating the channel unequally depending on the relative channel distributions in both TSR and PSR protocols.

\section{B. Performance Optimization and Impact of Log-normal Fad- ing Parameters}

In this section, we address the optimization problem of the EH time factor and the power-splitting factor for the TSR and PSR systems. In addition, we also discuss the impact of log-normal fading, i.e., the impact of the distribution parameters, on the behavior of the different protocols deployed in this work. In this respect, we show in Fig. 5 numerical

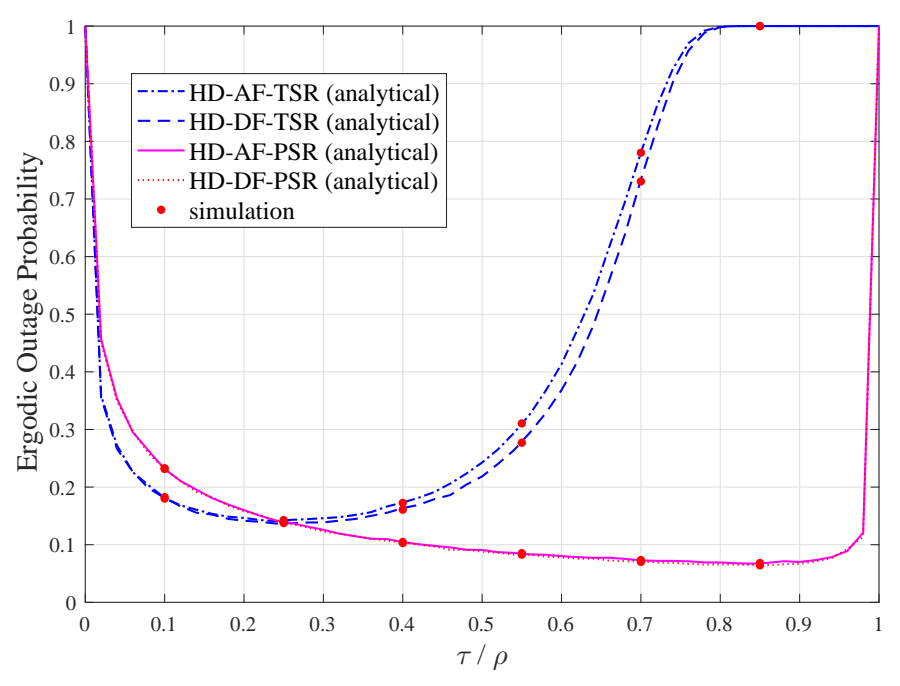

Figure 4: Ergodic outage probability performance with respect to $\tau$ and $\rho$ for the HD-DF-TSR, HD-DF-PSR, HD-AF-TSR and HD-AF-PSR systems.

results of the minimum achievable ergodic outage probability as a function of the source-to-relay and relay-to-destination channel variances for the HD-DF-TSR, HD-DF-PSR, HDAF-TSR and HD-AF-PSR systems with different transmit power values; these results are obtained using (19), (36), (60) and (65), respectively. Note that although it is very difficult to get the solution in closed-form, it does not pose any difficulty to obtain numerical solutions using software tools. For comparison's sake, results for the HD-DF-IRR and HDAF-IRR are also included on this figure, which are obtained from (50) and (69), respectively. A number of observations can be seen from this figure. For instance, as opposed to the Rayleigh fading case, it is observed that increasing the channel variance will always degrade performance for all the considered systems. Similar to the previous section, results in Fig. 5 indicate that DF-based systems always outperform the AF-based ones throughout the channel variance spectrum. It is also noticeable that the optimized PST schemes perform better than the optimized TSR systems and that the IRR system serves as a lower bound. Furthermore, comparing Figs. 5(a) and 5(b), it can be noted that increasing the source transmit power considerably enhances the ergodic outage probability for all the system configurations, which is intuitive.

\section{Impact of Processing and Channel Estimation Energy Cost}

Although the assumptions of perfect channel state information availability and the zero processing energy cost at 


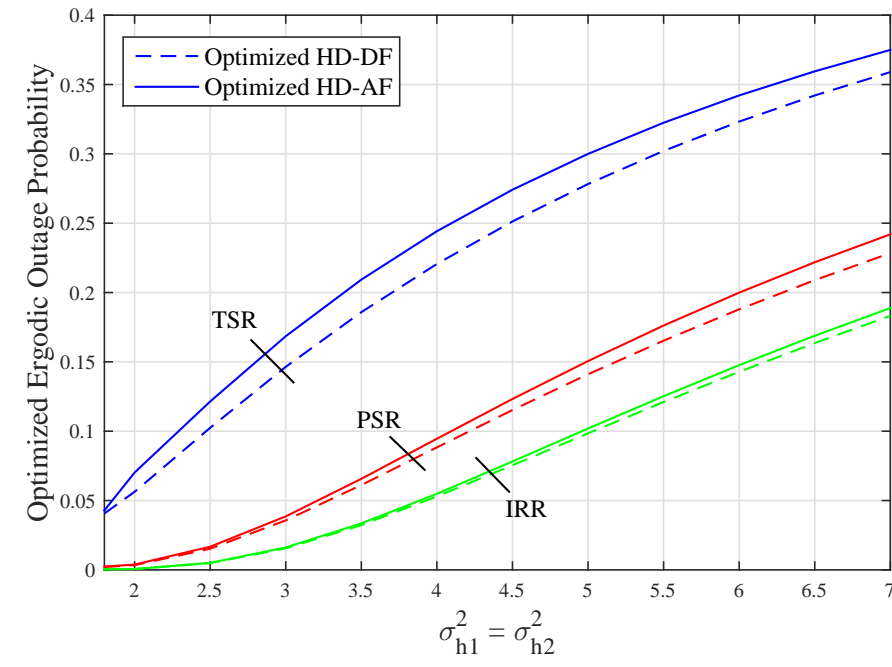

(a) $P_{s}=1 \mathrm{~W}$.

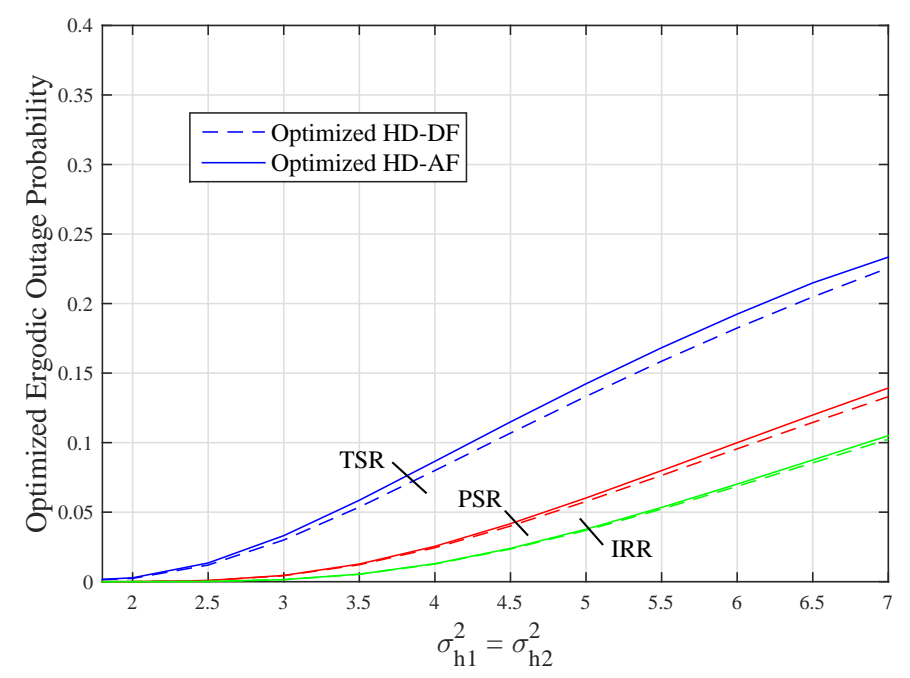

(b) $P_{s}=5 \mathrm{~W}$

Figure 5: Minimum achievable ergodic outage probability versus the source-to-relay and relay-to-destination channel variance for the HD-DFTSR, HD-DF-PSR, HD-DF-IRR, HD-AF-TSR, HD-AF-PSR and HDAF-IRR systems when $P_{s}=1 \mathrm{~W}$ and $5 \mathrm{~W}$.

the DF relay have simplified our analysis in this work, such assumptions are unrealistic in practice. We therefore dedicate this section to examine the impact of the energy cost at the DF relay due to information processing and channel estimation; the combined power cost will be referred to as $\left(P_{c}\right)$. The focus here will particularly be kept on the IRR protocol and the end-to-end distance is fixed at $30 \mathrm{~m}$. With this in mind, Fig. 6 demonstrates the ergodic outage probability for both the HD-AF-IRR and HD-DF-IRR with respect to the sourceto-relay distance with different values of $P_{c}$; specifically, we consider $P_{c}=0,0.01 P_{r}$, and $0.02 P_{r} \mathrm{~W}$, which correspond to $0 \%, 1 \%$ and $2 \%$ of the harvested power.

As one can readily observe from this figure, the ideal HDDF-IRR scheme, i.e., $P_{c}=0 \mathrm{~W}$, always outperforms the HDAF-IRR system regardless of the position of the relay. However, when the energy consumption at the DF relay is taken into account, the performance of both $\mathrm{DF}$ and $\mathrm{AF}$ relaying systems will become very comparable, e.g., $P_{c}=0.01 P_{r} \mathrm{~W}$.

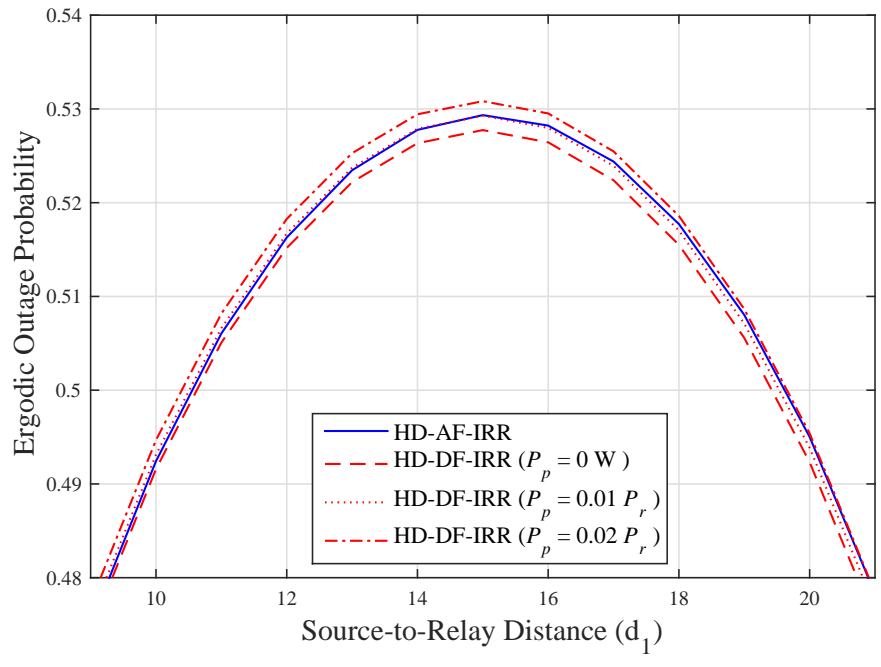

Figure 6: Ergodic outage probability versus the source-to-relay distance for the HD-DF-IRR and HD-AF-IRR systems with different values of $P_{c}$ at the DF relay when $d_{1}+d_{2}=30 \mathrm{~m}$.

However, when $P_{c}$ increases further, i.e., $P_{c}=0.02 P_{r} \mathrm{~W}$, AF relaying can start to perform better than DF relaying making the former more attractive in some practical scenarios. Another interesting remark on the results in Fig. 6 is that the poorest probability performance, for $\mathrm{AF}$ and $\mathrm{DF}$ relaying alike, is experienced when the relay is placed at the midpoint between the source and destination nodes. This is simply justified by the fact that at this point the relay will need more time harvesting energy and this consequently impacts the information transmission time; hence, high ergodic outage probability occurs.

\section{FD versus $H D$ Relaying}

The performance of the FD and HD systems with DF and AF relaying is discussed in this section. Fig. 7 depicts the ergodic outage probability as a function of the transmission rate threshold for the FD-DF and FD-AF systems with various values of the loop-back interference channel variance and the source transmit power; more specifically, $\sigma_{g}^{2}=\{2,5 \mathrm{~dB}\}$ and $P_{s}=\{1,10 \mathrm{~W}\}$. Results for the HD-DF and HD-AF schemes are also included in this figure. Note that the analytical results of the FD-DF and FD-AF systems are obtained from (81) and (92), respectively. The system parameters used in here are: $d_{1}=d_{2}=5 \mathrm{~m}, \tau=0.01, \sigma_{1}^{2}=\sigma_{2}^{2}=4 \mathrm{~dB}$ and $\mu_{1}=\mu_{2}=\mu_{g}=3 \mathrm{~dB}$. It is can be seen from Fig. 7(a) that when the loop-back interference channel variance is $\sigma_{g}^{2}=5 \mathrm{~dB}$, the FD schemes always outperform the HD ones for the same transmission rate irrespective of the value of $P_{s}$. On the other hand, however, it is demonstrated in Fig. 7(b) that when the loop-back interference channel variance $\sigma_{g}^{2}=2 \mathrm{~dB}$, HD outperforms FD. The final remark on these results is that FD-DF relaying has better performance than FD-AF relaying in all the protocols deployed.

\section{CONCLUSIONS}

This paper studied the ergodic outage probability of HD and FD relaying in EH networks over log-normal fading channels. 


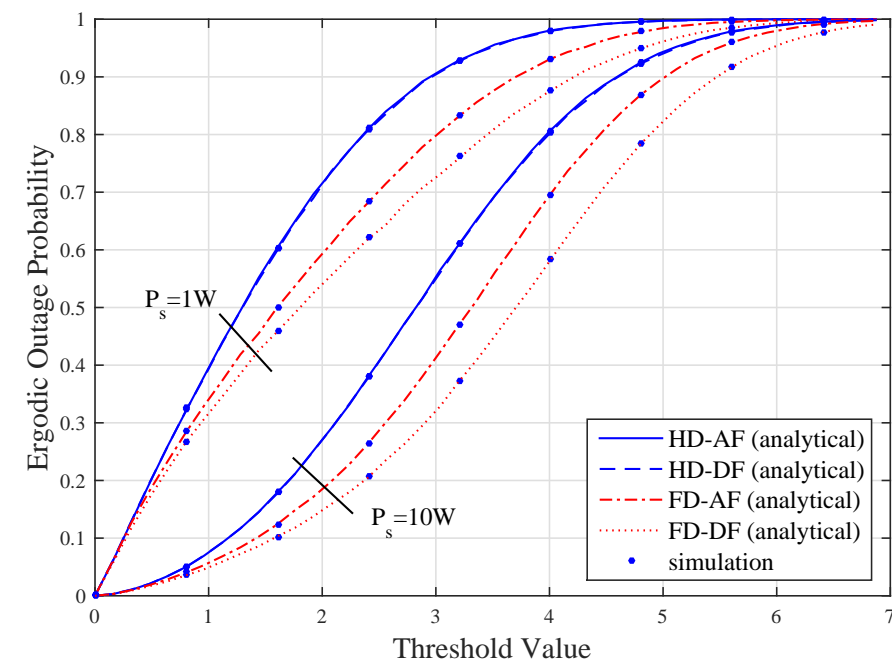

(a) $\sigma_{g}^{2}=5 \mathrm{~dB}$

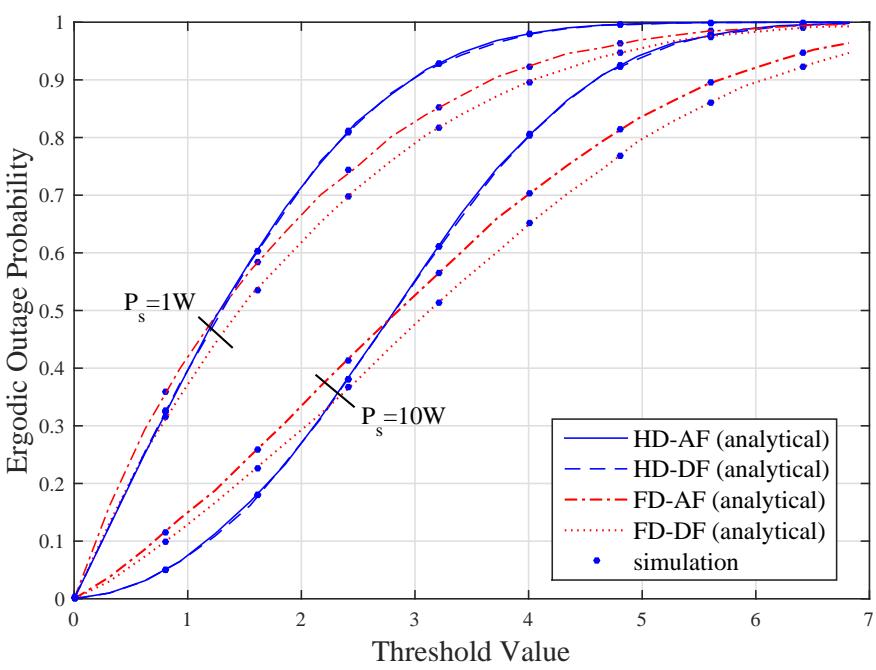

(b) $\sigma_{g}^{2}=2 \mathrm{~dB}$

Figure 7: Ergodic outage probability with respect to the transmission rate value for the FD-DF, FD-AF, HD-DF and HD-AF systems with different values of $\sigma_{g}^{2}$ and $P_{s}$. All results in this figure are based on the TSR protocol.

More specifically, we investigated the performance of both AF and DF relaying with three well-known EH protocols, namely, TSR, PSR and IRR. Accurate analytical expressions for the ergodic outage probability for these systems were derived and then validated with computer simulations. It was demonstrated that DF relaying is able to always offer better probability performance compared to AF relaying when the processing energy cost for the former at the relay is ignored. However, when the processing energy cost is taken into account $\mathrm{AF}$ relaying may outperform DF relaying. It was also shown that increasing the variance of the log-normal fading channel will degrade the performance. Comparing the performances of FD and HD relaying systems, it was found that FD relaying can considerably enhance the system performance as long as the loop-back interference due FD relaying is relatively low. However, if this interference increases, performance may severely degrade and consequently HD relaying can perform better. Finally, it is to be pointed out that a relay channel with the direct link will be a subject of future research.

\section{ACKNOWLEDGMENT}

This research has been carried out within the "Smart InBuilding Micro Grid for Energy Management" project funded by EPSRC (EP/M506758/1) and supported by Innovate UK (Innovate UK Project 101836).

\section{REFERENCES}

[1] L. Liu, R. Zhang, and K. C. Chua, "Wireless information transfer with opportunistic energy harvesting," IEEE Trans. Wireless Commun., vol. 12, pp. 288-300, Jan. 2013.

[2] J. Park and B. Clerckx, "Joint wireless information and energy transfer in a two-user MIMO interference channel," IEEE Trans. Wireless Commun., vol. 12, pp. 4210-4221, Aug. 2013.

[3] L. R. Varshney, "Transporting information and energy simultaneously," in IEEE Int. Symp. Inf. Theory, pp. 1612-1616, Jul. 2008.

[4] K. M. Rabie, B. Adebisi, and M. Rozman, "Outage probability analysis of wpt systems with multiple-antenna access point," in Int. Symp. Commun. Systems, Networks and Digital Signal Processing (CSNDSP), pp. 1-5, Jul. 2016.
[5] P. Grover and A. Sahai, "Shannon meets tesla: Wireless information and power transfer," in IEEE Int. Symp. Inf. Theory, pp. 2363-2367, Jun. 2010.

[6] A. M. Fouladgar and O. Simeone, "On the transfer of information and energy in multi-user systems," IEEE Commun. Lett., vol. 16, pp. 1733 1736, Nov. 2012.

[7] I. Krikidis, S. Timotheou, and S. Sasaki, "RF energy transfer for cooperative networks: Data relaying or energy harvesting?," IEEE Commun. Lett., vol. 16, pp. 1772-1775, Nov. 2012.

[8] A. Nasir, X. Zhou, S. Durrani, and R. Kennedy, "Relaying protocols for wireless energy harvesting and information processing," IEEE Trans. Wireless Commun., vol. 12, pp. 3622-3636, Jul. 2013.

[9] Z. Chen, B. Xia, and H. Liu, "Wireless information and power transfer in two-way amplify-and-forward relaying channels," in Proc. IEEE Global Conf. Signal and Inf. Process. (GlobalSIP), pp. 168-172, Atlanta, GA, Dec. 2014.

[10] A. A. Nasir, X. Zhou, S. Durrani, and R. A. Kennedy, "Throughput and ergodic capacity of wireless energy harvesting based DF relaying network," in Proc. IEEE Int. Conf. Commun (ICC), pp. 4066-4071, Jun. 2014.

[11] I. Krikidis, "Simultaneous information and energy transfer in largescale networks with/without relaying," IEEE Trans. Commun., vol. 62, pp. 900-912, Mar. 2014.

[12] A. A. Al-habob, A. M. Salhab, S. A. Zummo, and M. S. Alouini, "Multidestination cognitive radio relay network with SWIPT and multiple primary receivers," in IEEE Wireless Commun. and Networking Conf. (WCNC), pp. 1-6, Mar. 2017.

[13] P. Liu, S. Gazor, I. M. Kim, and D. I. Kim, "Energy harvesting noncoherent cooperative communications," IEEE Trans. Wireless Commun., vol. 14, pp. 6722-6737, Dec. 2015.

[14] P. K. Sharma and P. K. Upadhyay, "Cooperative spectrum sharing in two-way multi-user multi-relay networks," IET Commun., vol. 10, no. 1, pp. 111-121, 2016.

[15] D. Michalopoulos, H. Suraweera, and R. Schober, "Relay selection for simultaneous information transmission and wireless energy transfer: A tradeoff perspective," IEEE J. Sel. Areas Commun., vol. 33, pp. 15781594, Aug. 2015.

[16] A. Salem, K. A. Hamdi, and K. M. Rabie, "Physical layer security with $\mathrm{RF}$ energy harvesting in AF multi-antenna relaying networks," IEEE Trans. Commun., vol. 64, pp. 3025-3038, Jul. 2016.

[17] A. E. Shafie, D. Niyato, and N. Al-Dhahir, "Security of rechargeable energy-harvesting transmitters in wireless networks," IEEE Wireless Commun. Lett., vol. 5, pp. 384-387, Aug. 2016.

[18] T. M. Hoang, T. Q. Duong, N. S. Vo, and C. Kundu, "Physical layer security in cooperative energy harvesting networks with a friendly jammer," IEEE Wireless Commun. Lett., vol. 6, pp. 174-177, Apr. 2017.

[19] M. Zhang and Y. Liu, "Energy harvesting for physical-layer security in 
OFDMA networks," IEEE Trans. Inf. Forensics and Security, vol. 11, pp. 154-162, Jan. 2016.

[20] T. Riihonen, S. Werner, R. Wichman, and E. Z. B., "On the feasibility of full-duplex relaying in the presence of loop interference," in IEEE Workshop on Signal Processing Advances in Wireless Commun., pp. 275-279, Jun. 2009.

[21] B. P. Day, A. R. Margetts, D. W. Bliss, and P. Schniter, "Full-duplex MIMO relaying: Achievable rates under limited dynamic range," IEEE Journal Sel. Areas in Commun., vol. 30, pp. 1541-1553, Sept. 2012.

[22] L. Anttila, D. Korpi, V. Syrjala, and M. Valkama, "Cancellation of power amplifier induced nonlinear self-interference in full-duplex transceivers," in Asilomar Conference on Signals, Systems and Computers, pp. 11931198, Nov. 2013.

[23] M. M. Zhao, Y. Cai, Q. Shi, M. Hong, and B. Champagne, "Joint transceiver designs for full-duplex $k$-pair MIMO interference channel with SWIPT," IEEE Trans. Commun., vol. 65, pp. 890-905, Feb. 2017.

[24] H. Liu, K. J. Kim, K. S. Kwak, and H. V. Poor, "Power splittingbased swipt with decode-and-forward full-duplex relaying," IEEE Trans. Wireless Commun., vol. 15, pp. 7561-7577, Nov. 2016.

[25] H. Liu, K. J. Kim, K. S. Kwak, and H. V. Poor, "Qos-constrained relay control for full-duplex relaying with swipt," IEEE Trans. Wireless Commun., vol. 16, pp. 2936-2949, May 2017.

[26] D. Wang, R. Zhang, X. Cheng, and L. Yang, "Capacity-enhancing fullduplex relay networks based on power splitting (PS-)SWIPT," IEEE Trans. Veh. Technol., vol. PP, no. 99, pp. 1-1, 2016.

[27] Z. Wen, X. Liu, N. C. Beaulieu, R. Wang, and S. Wang, "Joint source and relay beamforming design for full-duplex MIMO AF relay SWIPT systems," IEEE Commun. Lett., vol. 20, pp. 320-323, Feb. 2016.

[28] C. Zhong, H. A. Suraweera, G. Zheng, I. Krikidis, and Z. Zhang, "Improving the throughput of wireless powered dual-hop systems with full duplex relaying," in IEEE Int. Conf. Commun. (ICC), pp. 4253 4258, Jun. 2015.

[29] K. M. Rabie, A. Salem, E. Alsusa, and M.-S. Alouini, "Energyharvesting in cooperative AF relaying networks over log-normal fading channels," in Proc. IEEE Int. Conf. Commun. (ICC), pp. 1-7, May 2016.

[30] F. Lotse, J.-E. Berg, and R. Bownds, "Indoor propagation measurements at $900 \mathrm{MHz}$," in Proc. Veh. Technol. Conf. (VTC), pp. 629-632, Denver, CO, May 1992.

[31] H. Hashemi, "The indoor radio propagation channel," IEEE Proc., vol. 81, pp. 943-968, Jul. 1993.

[32] S. Seidel, K. Takamizawa, and T. Rappaport, "Application of secondorder statistics for an indoor radio channel model," in Proc. Veh. Technol. Conf. (VTC), pp. 888-892, San Francisco, CA, May 1989.

[33] M.-S. Alouini and M. K. Simon, "Dual diversity over correlated $\log$ normal fading channels," IEEE Trans. Commun., vol. 50, pp. 1946-1959, Dec. 2002.

[34] M. D. Renzo, F. Graziosi, and F. Santucci, "A comprehensive framework for performance analysis of cooperative multi-hop wireless systems over log-normal fading channels," IEEE Trans. Commun., vol. 58, pp. 531544, Feb. 2010

[35] F. Heliot, X. Chu, R. Hoshyar, and R. Tafazolli, "A tight closed-form approximation of the log-normal fading channel capacity," IEEE Trans. Wireless Commun., vol. 8, pp. 2842-2847, Jun. 2009.

[36] M. Ghavami, L. Michael, and R. Kohno, Ultra Wideband Signals and Systems in Communication Engineering. Europe: Wiley, 2004.

[37] M. Safari and M. Uysal, "Cooperative diversity over log-normal fading channels: performance analysis and optimization," IEEE Trans. Wireless Commun., vol. 7, pp. 1963-1972, May 2008.

[38] A. Kalantari, M. Mohammadi, and M. Ardebilipour, "Performance analysis of opportunistic relaying over imperfect non-identical $\log$ normal fading channels," in Proc. IEEE Int. Symp. Personal, Indoor and Mobile Radio Commun. (PIMRC), pp. 1909-1913, Sept. 2011.

[39] C. Oestges, N. Czink, B. Bandemer, and A. Paulraj, "Capacity performance of outdoor-to-indoor relay schemes in measured radio channels," in IEEE Int. Symp. Personal, Indoor and Mobile Radio Commun., pp. 1113-1117, Sept. 2009.

[40] P. Kyritsi, P. Eggers, R. Gall, and J. M. Lourenco, "Measurement based investigation of cooperative relaying," in IEEE Veh. Technol. Conf. (VTC), pp. 1-5, Sept. 2006.

[41] K. M. Rabie, B. Adebisi, and M. S. Alouini, "Wireless power transfer in cooperative DF relaying networks with log-normal fading," in IEEE Global Commun. Conf. (GLOBECOM), pp. 1-6, Dec. 2016.

[42] S. J. Ambroziak, L. M. Correia, R. J. Katulski, M. Mackowiak, C. Oliveira, J. Sadowski, and K. Turbic, "An off-body channel model for body area networks in indoor environments," IEEE Trans. Antennas and Propagation, vol. 64, pp. 4022-4035, Sept. 2016.
[43] A. Laourine, A. Stephenne, and S. Affes, "Estimating the ergodic capacity of log-normal channels," IEEE Commun. Lett., vol. 11, pp. 568570, Jul. 2007.

[44] C. Zhong, H. A. Suraweera, G. Zheng, I. Krikidis, and Z. Zhang, "Wireless information and power transfer with full duplex relaying," IEEE Trans. Commun., vol. 62, pp. 3447-3461, Oct. 2014.

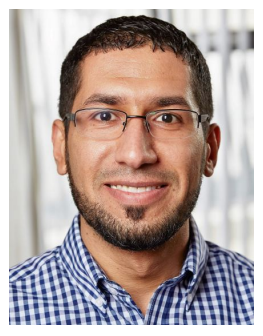

Khaled Maaiuf Rabie (SM'12, M'15), received the B.Sc. degree (with Hons.) from University of Tripoli, Libya, and the M.Sc. degree (with Hons.) from the university of Manchester, UK, in 2008 and 2010 , respectively. In 2011, he joined the university of Manchester where he worked as part-time staff and received his Ph.D. degree in Communication Engineering in 2015. He is currently a Postdoctoral Research Associate at Manchester Metropolitan University (MMU), Manchester, UK. His research interests include signal processing and analysis of powerline and wireless communication networks. Dr. Rabie received several awards, both nationally and internationally, including the Agilent Technologies' best M.Sc. student award, the Manchester Doctoral College Ph.D. scholarship and the MMU Outstanding Knowledge Exchange Project award of 2016. He was also the recipient of the best student paper award at the IEEE International Symposium on Power Line Communications and its applications (ISPLC) in 2015, Texas, US

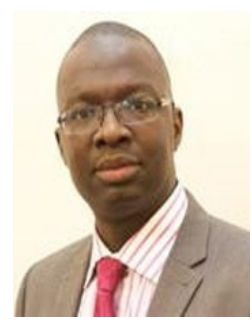

Bamidele Adebisi (M'06, SM'15) received his Master's degree in advanced mobile communication engineering and Ph.D. in communication systems from Lancaster University, UK, in 2003 and 2009, respectively. Before that, he obtained a Bachelor's degree in electrical engineering from Ahmadu Bello University, Zaria, Nigeria, in 1999. He was a senior research associate in the School of Computing and Communication, Lancaster University between 2005 and 2012. He joined Metropolitan University, Manchester in 2012 where he is currently a Reader in Electrical and Electronic Engineering. He has worked on several commercial and government projects focusing on various aspects of wireline and wireless communications. He is particularly interested in Research and Development of communication technologies for electrical energy monitoring/management, transport, water, critical infrastructures protection, home automation, IoTs and Cyber Physical Systems. He has several publications and a patent in the research area of data communications over power line networks and smart grid. He is a member of IET and a senior member of IEEE.

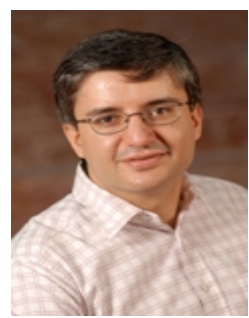

Mohamed-Slim Alouini (S'94, M'98, SM'03, F'09) was born in Tunis, Tunisia. He received the Ph.D. degree in Electrical Engineering from the California Institute of Technology (Caltech), Pasadena, CA, USA, in 1998. He served as a faculty member in the University of Minnesota, Minneapolis, MN USA, then in the Texas A \& M University at Qatar, Education City, Doha, Qatar before joining King Abdullah University of Science and Technology (KAUST), Thuwal, Makkah Province, Saudi Arabia as a Professor of Electrical Engineering in 2009. His current research interests include the modeling, design, and performance analysis of wireless communication systems. 